\title{
Contextual control over equivalence and nonequivalence explains apparent arbitrary applicable relational responding in accordance with sameness and opposition
}

\author{
Benigno Alonso-Álvarez ${ }^{1}$ • Luis Antonio Pérez-González ${ }^{1}$
}

Published online: 8 March 2017

(C) Psychonomic Society, Inc. 2017

\begin{abstract}
We evaluated whether contextual control over equivalence and nonequivalence (i.e., selecting comparisons equivalent to the samples in the presence of a contextual cue, and excluding the selection of comparisons equivalent to the samples in the presence of another contextual cue) can account for apparent arbitrarily applicable relational responding (AARR) in accordance with the frames of sameness and opposition, as defined in relational frame theory (RFT). Three college students were trained to maintain previously established conditional discriminations in the presence of a contextual cue $\mathrm{X} 1$, and to reverse them in the presence of another contextual cue X2 (e.g., X1-A1B1, X1-A2B2, X2A1B2, X2-A2B1). Subsequent tests demonstrated that X1 and $\mathrm{X} 2$ functioned as cues for equivalence and nonequivalence. Later on, X1 and X2 were demonstrated to be functionally equivalent to supposed contextual cues for the frames of sameness and opposition employed in RFT studies (i.e., SAME and OPPOSITE cues), in tests for arbitrary and nonarbitrary derived stimulus relations. The functional equivalence of X2 and OPPOSITE suggests that OPPOSITE worked as a cue for nonequivalence. Thus, the results in RFT studies with SAME and OPPOSITE can be explained just by contextual control over equivalence and nonequivalence. Therefore, the explanation that they actually demonstrated AARR in accordance with the frames of sameness and opposition can be questioned and replaced by a more parsimonious explanation,
\end{abstract}

Benigno Alonso-Álvarez

alonsobenigno@uniovi.es

Luis Antonio Pérez-González

laperez@uniovi.es

1 Department of Psychology, University of Oviedo, Despacho 209, Plaza Feijoo s/n, CP 33003 Oviedo, Spain based on a few simple learning principles. We discuss the implications of this conclusion for the debate among competing theories about the origin of stimulus equivalence and other derived stimulus-stimulus relations.

Keywords Contextual control - Stimulus equivalence · Exclusion $\cdot$ Frame of opposition $\cdot$ Relational frame theory

Relational frame theory (RFT) is a modern behavioral account of human language and cognition (e.g., Hayes, Barnes-Holmes, \& Roche, 2001), based on the concept of arbitrary applicable relational responding (AARR). Relational responding means responding to one stimulus in terms of another, but, in contrast with the more familiar forms of nonarbitrary relational responding (NARR), such as generalized identity or oddity matching, AARR does not depend on the physical properties of the stimuli related; AARR would be "brought to bear" by relational cues or contexts (Hayes et al., 2001, p. 32). The RFT proposes the existence of specific types of AARR or relational frames, such as the relational frames of sameness, opposition, distinction, and so forth, defined by specific properties of mutual entailment (e.g., if $\mathrm{A}$ is the same as B, B is the same of $\mathrm{A}$ ), combinatorial entailment (e.g., if $\mathrm{A}$ is the opposite of $\mathrm{B}$, and $\mathrm{B}$ is the opposite of $\mathrm{C}$, then $\mathrm{A}$ is the same as $\mathrm{C}$ ), and transformation of functions (e.g., if A is the same as $\mathrm{C}$, and $\mathrm{C}$ is an excitatory conditioned stimulus, then A will also be an excitatory stimulus without the need of direct conditioning). RFT hypothesizes that relational frames are the outcome of relational training histories across multiple exemplars and are thus conceptualized as generalized, higher order, or overarching operant classes (Hayes et al., 2001, p. 23). 
AARR in accordance with the frames of sameness and opposition are two of the types of AARR most frequently investigated in the RFT literature (e.g., Bennett, Hermans, Dymond, Vervoort, \& Baeyens, 2015; Cassidy, Roche, \& Hayes, 2011; Dymond, Roche, Forsyth, Whelan, \& Rhoden 2007, 2008; Dymond, Tsz, \& Whelan, 2013; Dymond \& Whelan, 2010; Ninness et al., 2009; Perez, de Almeida, \& de Rose, 2015; Roche \& Barnes, 1996, 1997; Roche, Barnes-Holmes, Smeets, Barnes-Holmes, \& McGeady, 2000; Steele \& Hayes, 1991; Stewart et al., 2015; Whelan \& Barnes-Holmes, 2004). In these studies, the typical procedure was as follows. First, participants were trained in second-order conditional discriminations involving nonarbitrary stimulus relations (see Fig. 1). The comparisons presented were three stimuli ordered along some physical dimension, like length or size (e.g., a short line, a medium line, a long line). The samples were two stimuli identical to the comparisons located at the extremes of the physical dimension along which the comparisons were ordered (e.g., a short line, and a long line). In the presence of a stimulus arbitrarily designated as a contextual cue for the relation of sameness (the SAME stimulus), participants were trained to select comparisons identical to the samples (e.g., choosing a short line comparison when a short line was the sample, and choosing a long line comparison when a long line was the sample). In the presence of another stimulus arbitrarily designated as contextual cue for the relation of opposition (the OPPOSITE stimulus), participants were trained to select comparisons the most physically dissimilar to the samples (e.g., choosing a short line comparison when a long line was the sample, and choosing a long line comparison when a short line was the sample). This training was repeated across multiple exemplars (e.g., small to large squares, sets of few to many dots) in the presence of SAME and OPPOSITE, and continued until participants consistently selected comparisons identical to the samples in the presence of SAME, and comparisons the most dissimilar to the samples in the presence of OPPOSITE, with new exemplars not used in training.
Next, participants were trained in second-order conditional discriminations involving arbitrary stimulus relations in the presence of SAME and OPPOSITE (see Fig. 2, left panel). They were trained to select comparisons $\mathrm{B} 1$ and $\mathrm{C} 1$ in the presence of sample $\mathrm{A} 1$ and SAME, and to select comparisons $\mathrm{B} 2$ and $\mathrm{C} 2$ in the presence of the sample $\mathrm{A} 1$ and OPPOSITE. Participants were then presented with tests for combinatorial entailment. In these tests, they typically selected $\mathrm{C} 1$ in the presence of $\mathrm{B} 1$ and SAME, $\mathrm{C} 2$ in the presence of $\mathrm{B} 2$ and SAME, $\mathrm{C} 2$ in the presence of $\mathrm{B} 1$ and OPPOSITE, and $\mathrm{C} 1$ in the presence of B2 and OPPOSITE (see Fig. 2, left panel).

RFT proponents interpreted these outcomes as AARR in accordance with the frames of sameness and opposition (e.g., Steele \& Hayes, 1991). The selection of $\mathrm{C} 1$ in the presence of $\mathrm{B} 1$ and SAME is consistent with the combinatorial entailment of two relations of sameness because, "the same of the same is the same" (e.g., If B1 is the same as A1, and $\mathrm{C} 1$ is the same as $\mathrm{A} 1$, then $\mathrm{C} 1$ is the same as $\mathrm{B} 1$ ). The selection of $\mathrm{C} 2$ in the presence of $\mathrm{B} 1$ and OPOSSITE, and the selection of $\mathrm{C} 1$ in the presence of $\mathrm{B} 2$ and OPPOSITE, is consistent with the combinatorial entailment of two relations of sameness and opposition because "the same of the opposite is the opposite" (e.g., if B1 is the same as $\mathrm{A} 1$, and $\mathrm{C} 2$ is the opposite of $\mathrm{A} 1$, then $\mathrm{C} 2$ is the opposite of B1). Finally, the selection of $\mathrm{C} 2$ in the presence of $\mathrm{B} 2$ and SAME is consistent with the combinatorial entailment of two relations of opposition because "the opposite of the opposite is the same" (i.e., if B2 is the opposite of A1, and $\mathrm{C} 2$ is the opposite of $\mathrm{A} 1$, then $\mathrm{C} 2$ is the same as $\mathrm{B} 2$ ). This account is supported by the outcomes of control participants who did not receive nonarbitrary relational training prior to the arbitrary relational training (see Fig. 2, right panel). These control participants, for whom SAME and OPPOSITE were only arbitrary cues, did not demonstrate AARR in accordance with the frames of sameness and opposition. For instance, they did not relate $\mathrm{C} 2$ and $\mathrm{B} 2$ in the presence of SAME (e.g., Steele \& Hayes, 1991).
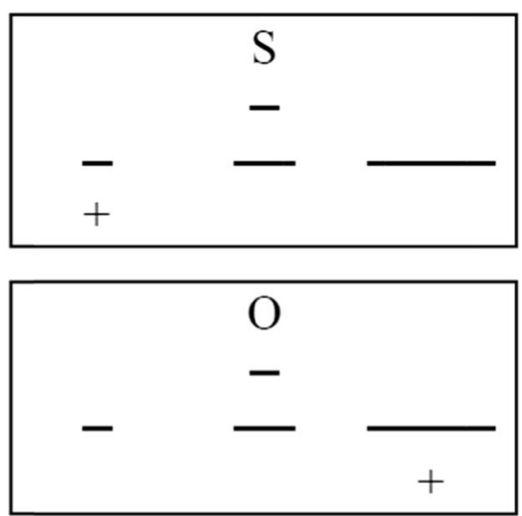

Fig. 1 Example of a discrimination involving NARR trained by Steel and Hayes (1991) and other studies that replicated this one. Contextual cues are represented at the top of each panel, samples in the middle, and
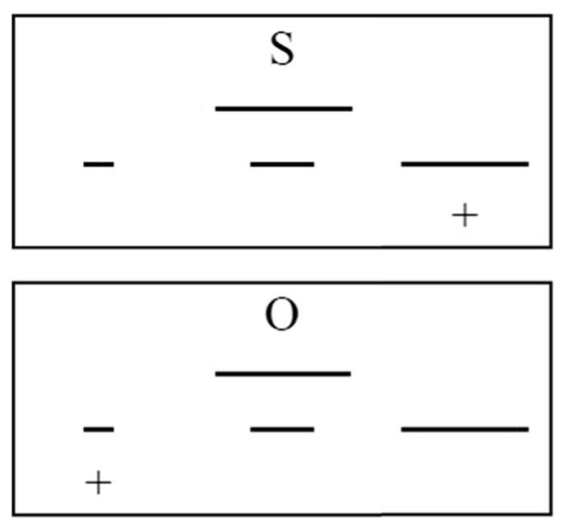

comparisons at the bottom. The $S$ and $O$ letters represent arbitrary stimuli trained as cues for sameness $(\mathrm{S})$ and opposition $(\mathrm{O})$. The plus symbol is placed below correct comparisons 


\section{Participants with previous NARR}

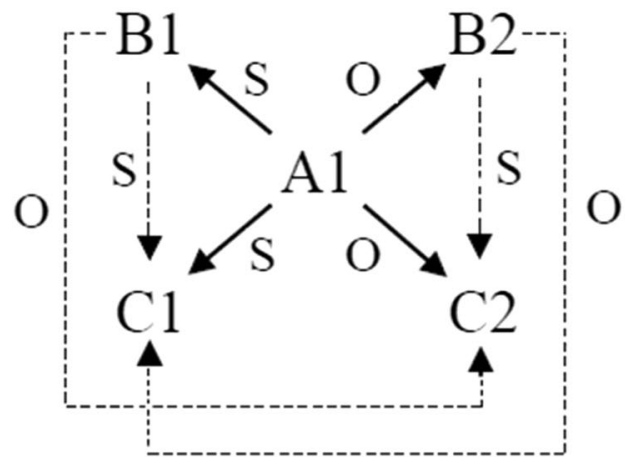

Fig. 2 Arbitrary stimulus relations demonstrated by participants in RFT studies on the frames of sameness and opposition. Arrows point from samples to comparisons. Solid-line arrows represent trained relations and dashed-line arrows represent derived relations. Symbols next to the arrows represent the contextual cues in whose presence the stimulus relations occurred. The left panel shows the stimulus relations

Apparent AARR in accordance with sameness and opposition, however, could be explained by alternative processes (as recognized by Steele \& Hayes, 1991, pp. 543, 552-553). These are the establishment of stimulus equivalence (SE) classes, responding based on exclusion of equivalence, and contextual control over responding based on equivalence and the exclusion of equivalence. SE is demonstrated when humans acquire - usually via matching-to-sample procedures - two or more overlapping conditional discriminations involving arbitrary stimulus relations (e.g., $\mathrm{AB} \& \mathrm{AC}$ ), and then they pass conditional discriminations tests for reflexivity (e.g., AA, BB, \& CC), symmetry (e.g., BA \& CA), and transitivity (e.g., BC $\&$ CB) - the defining properties of equivalence relations. Positive outcomes in these tests demonstrate the formation of SE classes (e.g., classes A1B1C1 \& A2B2C2; e.g., Sidman, 1994). According to Sidman's (2000) theory, SE occurs as a direct outcome of the operant contingencies of reinforcement applied during conditional discrimination training (SE and AARR in accordance with sameness refer to the same phenomenon, but Sidman and the RFT hypothesize that it occurs as a result of different processes).

Responding by exclusion in conditional discriminations occur when participants familiar with matching-to-sample are presented with a familiar sample, a comparison whose selection was punished in the presence of that sample and a novel comparison, and the participants select the novel comparison. When SE comes into play, this paradigm may be expanded and participants presented with a sample from a given SE class, a comparison from a different SE class and a novel comparison, select the novel comparison (i.e., they exclude the selection of the comparison nonequivalent to the sample; e.g., de Rose, de Souza, \& Hanna, 1996; Stromer \& Osborne, 1982).

\section{Participants without NARR}

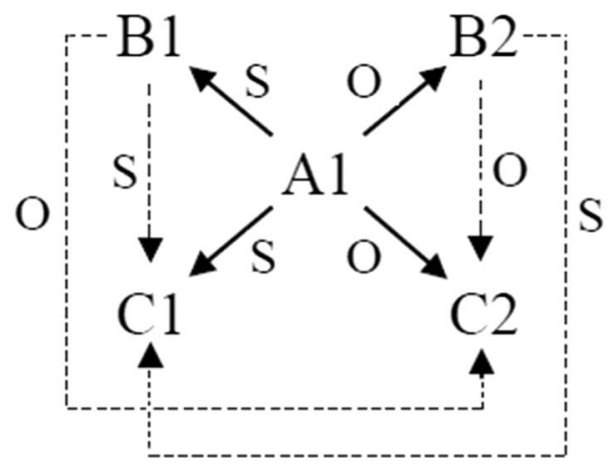

demonstrated by participants who had been presented with a previous NARR phase, where two arbitrary stimuli were pretrained as cues for sameness (S) and opposition (O). The right panel shows the relations demonstrated by participants who had not been presented with a previous NARR phase

Contextual control over equivalence occurs when participants select comparisons equivalent to the samples in the presence of a given contextual stimulus and exclude the selection of comparisons equivalent to the samples in the presence of a different contextual stimulus (e.g., Lynch \& Green, 1991; Pérez-González \& Serna, 2003; Steele \& Hayes, 1991). This process implies the establishment of second-order conditional discriminations, where the conditional relations among samples and comparisons are themselves subject to conditional control that is exerted by contextual stimuli (Sidman, 1986). The formation of SE classes, responding based on the exclusion of equivalence and contextual control over equivalence, will be referred hereafter as contextual control over equivalence and nonequivalence, where nonequivalence means exclusion of equivalence.

Contextual control over equivalence and nonequivalence can explain apparent AARR in accordance with the frames of sameness and opposition as follows: Following the NARR phase, the cue for the selection of comparisons identical to the samples (SAME) could become a cue for selecting comparisons equivalent to the samples because identical stimuli are equivalent stimuli (e.g., Sidman, 1994). In addition, the contextual cue for the selection of comparisons the most dissimilar to the samples (OPPOSITE) could become a cue for the exclusion of comparisons equivalent to the samples (i.e., nonequivalent comparisons). Consequently, after training participants to select $\mathrm{B} 1$ or $\mathrm{C} 1$ in the presence of $\mathrm{A} 1$ and SAME, the stimuli A1, B1 and C1 would become equivalent. Thus, during tests participants would have selected $\mathrm{C} 1$ in the presence of $\mathrm{B} 1$ and SAME because $\mathrm{C} 1$ and $\mathrm{B} 1$ were equivalent. In addition, training participants to select $\mathrm{B} 2$ and $\mathrm{C} 2$ in the presence of A1 and OPPOSITE would have made of B2 and C2 stimuli nonequivalent to A1 (i.e., correct choices in the 
presence of a cue for the exclusion of equivalence, and A1). $\mathrm{As} \mathrm{B} 1$ and $\mathrm{C} 1$ would be equivalent to $\mathrm{A} 1$, then $\mathrm{B} 2$ and $\mathrm{C} 2$ would also be nonequivalent to $\mathrm{B} 1$ and $\mathrm{C} 1$. Thus, participants would have selected $\mathrm{C} 1$ in the presence of $\mathrm{B} 2$ and OPPOSITE because $\mathrm{C} 1$ and $\mathrm{B} 2$ were nonequivalent. Likewise, participants would have selected $\mathrm{C} 2$ in the presence of $\mathrm{B} 1$ and OPPOSITE because C2 and B1 were nonequivalent. Finally, participants would have selected $\mathrm{C} 2$ in the presence of $\mathrm{B} 2$ and SAME by the exclusion of $\mathrm{C} 1$. If $\mathrm{C} 1$ and $\mathrm{B} 2$ were nonequivalent, then $\mathrm{C} 1$ could not have been the correct choice in the presence $\mathrm{B} 2$ and a cue for equivalence (SAME). Thus, participants excluded $\mathrm{C} 1$ and selected $\mathrm{C} 2$.

Determining whether contextual control over equivalence and nonequivalence can account for AARR in accordance with the frames of sameness and opposition has important implications for the current debate among the different theories about the origin of derived stimulus relations (e.g., Clayton \& Hayes, 1999). RFT proponents argue that their theory is "the only account of relational activity that explains response patterns in accordance with relations other than equivalence" (Roche et al., 2000, p. 268), and thus it has an edge over alternative theories of derived stimulus-stimulus that are focused only on equivalence (e.g., Horne \& Lowe, 1996; Sidman, 2000). Their claims would be contradicted if it were demonstrated that apparent AARR in accordance with relations other that equivalence can be explained by alternative processes to those proposed by the RFT.

In this research, we evaluated whether apparent AARR in accordance with sameness and opposition can be explained by contextual control over equivalence and nonequivalence. Our general strategy consisted of demonstrating that two stimuli pretrained as contextual cues for equivalence and nonequivalence - instead of for sameness and opposition - can control the same type of derived responding as SAME and OPPOSITE did in RFT studies. In Phase 1, a stimulus X1 was pretrained as a cue for maintaining previously trained conditional discriminations, and a stimulus X2 was pretrained as a cue for reversing those discriminations (Pérez-González, Álvarez, Calleja, \& Fernández, 2014; Pérez-González, Díaz, Fernández-García, \& Baizán, 2015; Pérez-González \& Martínez, 2007; Pérez-González \& Serna, 2003; Serna \& Pérez-González, 2003). This pretraining potentially makes of $\mathrm{X} 1$ and $\mathrm{X} 2$ cues for equivalence and nonequivalence (see Pérez-González et al., 2014; Pérez-González \& Serna, 2003). In Phase 2, we aimed to confirm whether $\mathrm{X} 1$ and $\mathrm{X} 2$ became cues for equivalence and nonequivalence, as a result of the pretraining of Phase 1. In Phases 3 and 4, we tested whether X1 and X2 controlled the same types of derived responding as SAME and OPPOSITE in RFT tests that supposedly evaluated combinatorial entailment and transformation of functions in accordance with the frames of sameness and opposition. In Phase 5, we evaluated whether SAME and X1, and OPPOSITE and $\mathrm{X} 2$, were functionally interchangeable for one another as it would be expected if, as we claim, X1 and SAME are both cues for equivalence, and $\mathrm{X} 2$ and OPPOSITE are both cues for nonequivalence. Positive outcomes in all the tests presented would demonstrate that apparent AARR in accordance with sameness and opposition in RFT studies can be explained by contextual control over equivalence and nonequivalence.

\section{Method}

\section{Participants}

Three psychology undergraduate students volunteered for participating in the experiment. They were recruited through an in class announcement and were compensated for their participation with 2 euros (about 2.3 U.S. dollars) per each $15 \mathrm{mi}-$ nutes of experimental session completed. All participants were Spanish speakers and were 18 years of age. They were designated as P101 (male), P102 (female), and P103 (female). They were unfamiliar with derived stimulus relations research.

\section{Apparatus and stimuli}

Sessions were conducted individually in an experimental room equipped with a table, a chair, and a laptop computer programmed in Visual Basic ${ }^{\circledR}$ for presenting matchingto-sample and simple discrimination trials. In matchingto-sample trials, the entire area of the computer's screen $(23 \mathrm{~cm} \times 30 \mathrm{~cm}$ in size $)$ displayed contextual cues, samples and comparisons arranged in an inverted " $\mathrm{T}$ " shape: Contextual cues were displayed at the top-center (their center was $8.6 \mathrm{~cm}$ below the top edge of the screen), samples at the middle-center (their center was $5.8 \mathrm{~cm}$ below the center of contextual cues), and comparisons at the bottom-center (their center was $5.8 \mathrm{~cm}$ below the center of samples), bottom-left, and bottom-right sections of the screen (the distance from center to center of the three comparisons was $7.4 \mathrm{~cm}$ ). Participants entered their responses by mouse clicking on the comparisons. Clicks were registered by the computer when they hit any of the invisible response fields $(3.7 \mathrm{~cm} \times 3.7 \mathrm{~cm}$ in size) where the comparisons were centered. Clicks outside these response fields had no effects. The computer's keyboard was deactivated.

In simple discrimination trials, the computer displayed contextual cues at the top center of the screen and discriminative stimuli at the middle center, exactly at the same locations as contextual cues and samples in matching-to-sample trials. 
Participants responded by pressing the space bar of the computer keyboard and then pressing the "enter" key. The computer mouse was deactivated. In these trials, the computer also displayed a counter that went up for one unit after each bar press. The size of the counter was $1.7 \mathrm{~cm} \times 1.6 \mathrm{~cm}$, it had its center $4.8 \mathrm{~cm}$ below the center of the discriminative stimuli and displayed black numbers in sans serif font, 36-point type size. Moreover, in some trials, textual prompts appeared immediately below the counter in black color and sans serif font, 12-point type size.

The computer presented visual feedback after some responses. Feedback consisted of the word Correct, displayed in blue color, or the word Error, displayed in red color (both words in Spanish). These words appeared at the center of the screen, in sans serif font, 27.7- point type size. Figure 3 shows all stimuli presented to participants. The stimuli, $3 \mathrm{~cm} \times 3 \mathrm{~cm}$ in size, were displayed in black color against a white background.

\section{Procedure}

Sessions and instructions. At the beginning of the experiment, participants sat in front of the computer and read the following instructions displayed on the screen, in Spanish:

Thank you for participating in this study. Next, several figures will appear at the top and bottom sections of the screen. Your task is choosing one of the figures displayed at the bottom of the screen using the computer mouse, after paying attention to the figures displayed at the top. Sometimes, the computer will let you know if you selected the right figure (you will see the words "Correct" or "Error" on the screen after your choice). In other occasions, the computer will not let you know if your choice was the right one, but try always to respond correctly. Click on the button below to begin the experiment.

The experimenter (the first author) clarified any fragment of the instructions if needed, but he did not add any

\section{Contextual Cues}

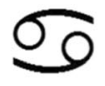

X1

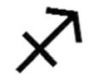

$\mathrm{X} 2$

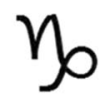

SAME

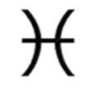

OPPOSITE
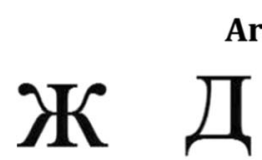

Arbitrary Relations

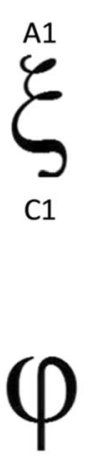

E1
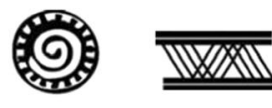

A2

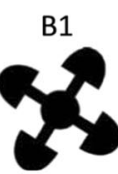

D1
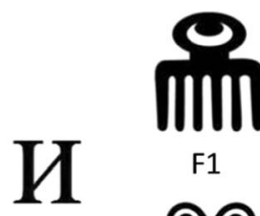

E2
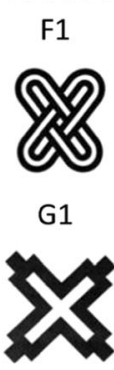

I1

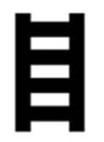

J1

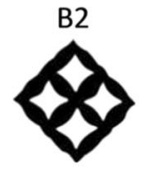

D2

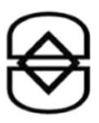

F2
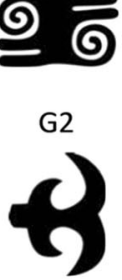

12

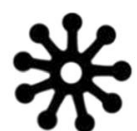

J2
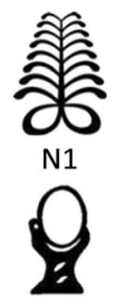

N2

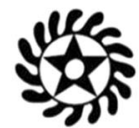

N3

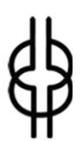

N4

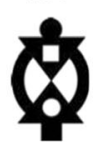

N5

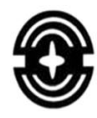

N6
Nonarbitrary Relations

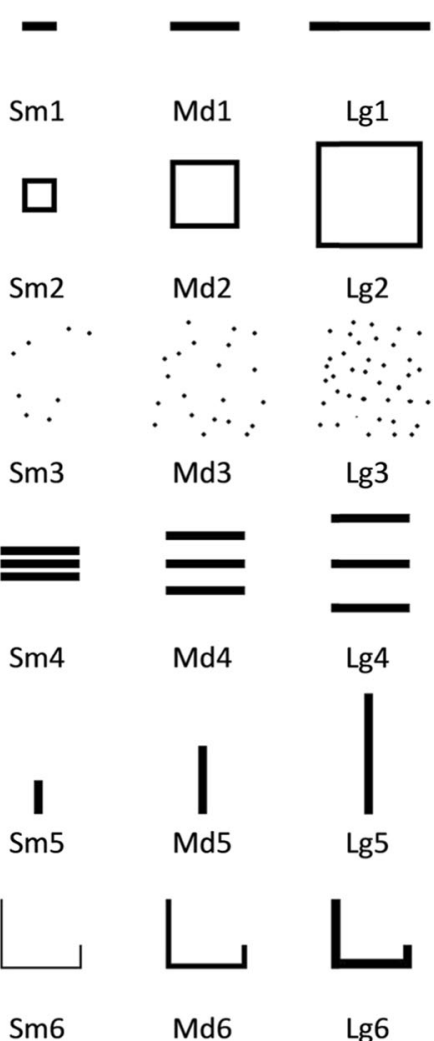

Fig. 3 Stimuli presented to the participants together with their alphanumeric designation (unbeknown for the participants). The expression "Sm" stands for small, "Md" stands for medium, and "Lg" stands for large 
supplementary information. Thereafter, he left the room and the experimental sessions started. Participants completed the experiment in a single session, which lasted 42 minutes for P101, 38 minutes for P102, and 30 minutes for P103. In the case of participant $\mathrm{P} 101$, the experimenter returned to the experimental room after the completion of each phase of the experiment (see below), asked the participant to leave the room momentarily, evaluated his performance, and set the computer to continue the experiment. Participants P102 and P103 received a slightly different version of the computer program that did not require the experimenter to enter until the end of the session. Once the experiment was over, the participants were asked to abstain from communicating to their classmates any details regarding the experiment.

Matching-to-sample trials. Matching-to-sample trials started with the simultaneous presentation of one sample and three comparisons, in the case of first-order conditional discriminations, or one contextual cue, one sample, and three comparisons, in the case of second-order conditional discriminations. The mouse cursor appeared simultaneously with the stimuli ( $2.7 \mathrm{~cm}$ above the bottom edge of the screen, and equidistant from its lateral edges). When participants clicked on a response field containing a comparison, all stimuli and the mouse cursor were cleared from the screen. In training trials, responses were followed by differential consequences. The word Correct followed correct responses, and the word Error followed incorrect responses. After 1.25 seconds, the feedback was cleared from the screen and the next trial started automatically. In test trials, participants' responses emptied the screen and next trial started automatically after $1.25 \mathrm{sec}-$ onds. In some trials, we used a prompting procedure that consisted of presenting the comparison designated as the correct choice as the only option available.

Simple discrimination trials. Simple discrimination trials started with the presentation of a discriminative stimulus, accompanied in some trials by a contextual cue. Both stimuli appeared simultaneously. The counter displayed beneath the discriminative stimuli started at zero in each trial and went up for one unit each time the participants pressed the space bar. We used a prompting procedure in some trials presented early in training. In these prompted trials, the computer displayed on the screen textual prompts instructing participants (in Spanish) to press the space bar twice or five times and then pressing the "enter" key. When participants pressed the "enter" key, all stimuli including the counter and textual prompts were removed from the screen. In training trials, participants' responses were followed by the words Correct or Error, depending on whether they were correct or not. After 1.25 seconds, the next trial started. In test trials, participants' responses were only followed by an intertrial interval of 1.25 seconds and the next trial.
Blocks of trials. Each phase of the experiment consisted of a sequence of trial blocks. Each block included several trial types defined by the antecedent stimuli presented in the trial (see Figs. 4, 5 and 6). The trial types included in a block appeared equally often in that block. On matching-to-sample blocks, the comparisons presented appeared equally often in each comparison location, both as correct and as incorrect choices (the only exception occurred in Block 1 of Phase 5, where only three out of four trial types appeared once per block as prompted trials; this happened because the computer had been programmed to present only three prompts).

The computer presented the trials included in a block in random order. In training blocks, the presentation of trials recommenced when participants made an error (i.e., they selected an incorrect comparison). The computer determined a new random order for the presentation of trials each time the block recommenced. Training blocks recommenced in this manner until participants responded to all trials from the block without errors. Thus, the number of trials programmed for a block was the learning criterion for that block (e.g., if a block included 12 trials, then the learning criterion for that block was 12 correct responses out of 12 trials). In tests blocks, the computer presented all the trials from the blocks without interruption, regardless of the participants' performance. We adopted a test criterion for deciding whether participants passed a test block or not. In some occasions, the achievement of this criterion determined if a sequence of training and test blocks was repeated or not.

\section{Phases}

Phase 1: $X 1$ and $X 2$ as contextual stimuli. In Phase 1, we intended to establish $\mathrm{X} 1$ and $\mathrm{X} 2$ as generalized contextual cues for maintaining and reversing conditional discriminations that had been previously trained in the absence of contextual cues. Phase 1 consisted of training the $\mathrm{AB}, \mathrm{XAB}$, and $\mathrm{CD}$ discriminations, followed by the XCD test (see Fig. 4, top panel). In these conditional discriminations - and in all others trained or tested in our experiment, see below-we presented a third $\mathrm{N}$ comparison in each trial as is customary in RFT studies on sameness and opposition. In the $\mathrm{AB}$ discrimination, the arbitrary stimulus relations trained were $\mathrm{A} 1 \mathrm{~B} 1$ and $\mathrm{A} 2 \mathrm{~B} 2$ (choosing N1 was never correct). In the XAB discrimination, the correct responses consisted of maintaining the stimulus relations trained in $\mathrm{AB}$, in the presence of $\mathrm{X} 1$, and reversing those relations, in the presence of $\mathrm{X} 2$ (i.e., the correct relations were $\mathrm{A} 1 \mathrm{~B} 1$ and $\mathrm{A} 2 \mathrm{~B} 2$ in the presence of $\mathrm{X} 1$, and $\mathrm{A} 1 \mathrm{~B} 2$ and $\mathrm{A} 2 \mathrm{~B} 1$ in the presence of $\mathrm{X} 2$ ).

In the $\mathrm{CD}$ discrimination, the relations trained were $\mathrm{C} 1 \mathrm{D} 1$ and C2D2 (choosing N2 was never correct). In the XCD test, the correct responses consisted of maintaining the stimulus relations trained in $\mathrm{CD}$, in the presence of $\mathrm{X} 1$, and reversing those 


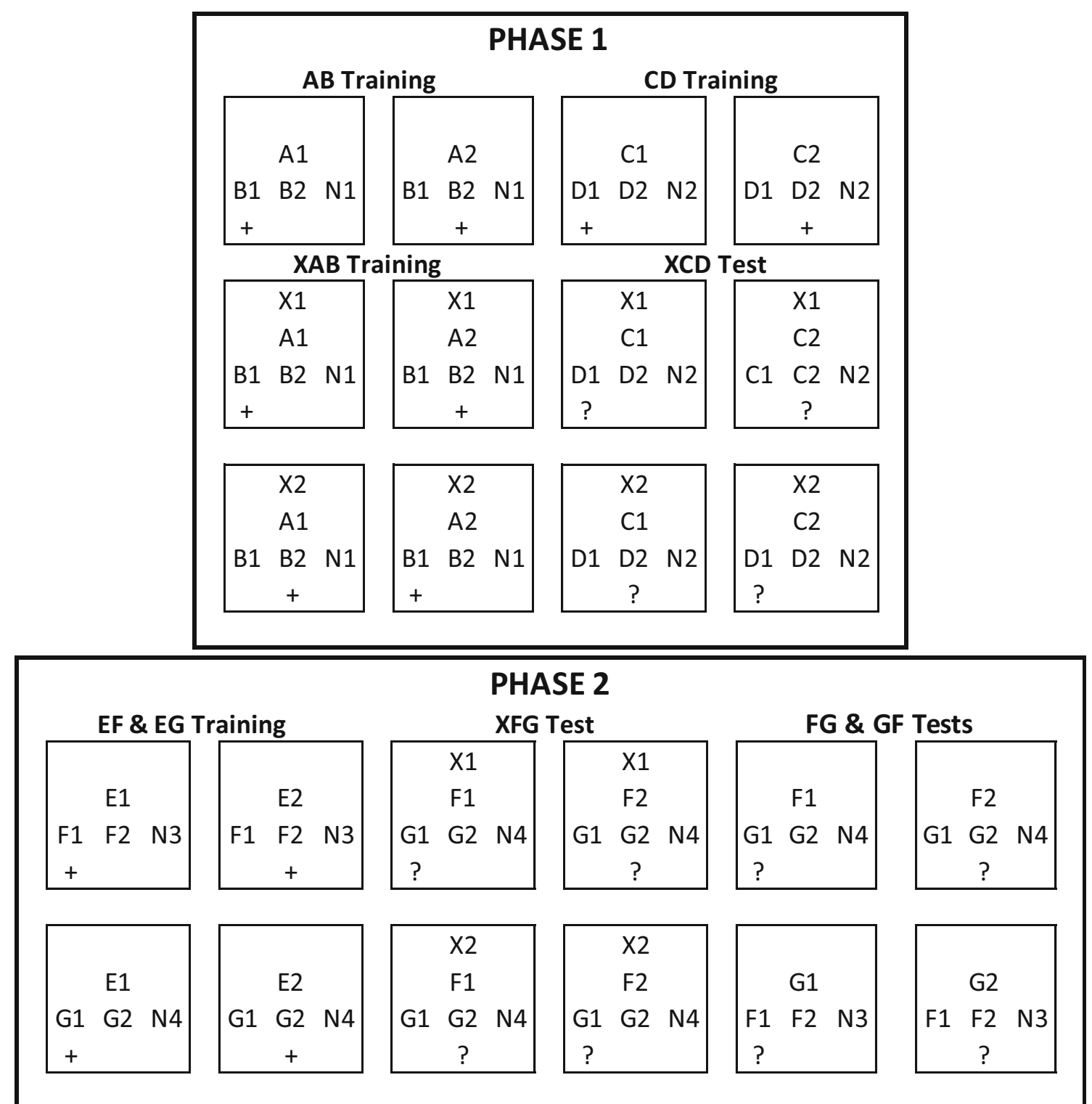

Fig. 4 Discriminations trained and tested in Phases 1 and 2. Each inner panel corresponds to a trial type from a discrimination. Contextual cues are represented at the top of each panel, samples in the middle, and

relations, in the presence of $\mathrm{X} 2$ (i.e., the correct relations were C1D1 and C2D2 in the presence of X1, and C1D2 and C2D1 in the presence of X2). Positive outcomes in this test would demonstrate that $\mathrm{X} 1$ and $\mathrm{X} 2$ became generalized cues for maintaining and reversing previously established arbitrary stimulus relations.

The sequence of blocks presented in Phase 1, the discriminations trained or tested in each block, and the number of trials per block appear in Tables 1, 2 and 3. Blocks 1 and 2 were presented to facilitate the training of $\mathrm{AB}$, which properly occurred in Block 3. Blocks 4 and 5 were presented to facilitate the training of $\mathrm{XAB}$, which properly occurred in Block 6 . When participants achieved the criterion for passing the XCD test (12 correct responses out of 12 trials), they moved on to comparisons at the bottom. Correct choices for each trial are indicated by a plus symbol, for correct choices followed by consequences, or by a question mark, for correct choices not followed by consequences

Phase 2. Otherwise, the training-probing sequence of Phase 1 recommenced: P101 moved back to Block 1, and P102 and P103 moved back to Block 3 (they were presented with different versions of the computer program used in the experiment). We had scheduled to repeat this sequence a maximum of four times.

Phase 2: $\mathrm{X} 1$ and $\mathrm{X} 2$ as cues for equivalence and nonequivalence. In Phase 2, we evaluated whether X1 and X2 functioned as cues for equivalence and nonequivalence as a result of the training provided in Phase 1. Phase 2 consisted of training the $\mathrm{EF}$ and $\mathrm{EG}$ discriminations, followed by the XFG, EF, and EG tests (see Fig. 4, lower panel). In the EF discrimination, the relations trained were E1F1 and E2F2 (choosing 

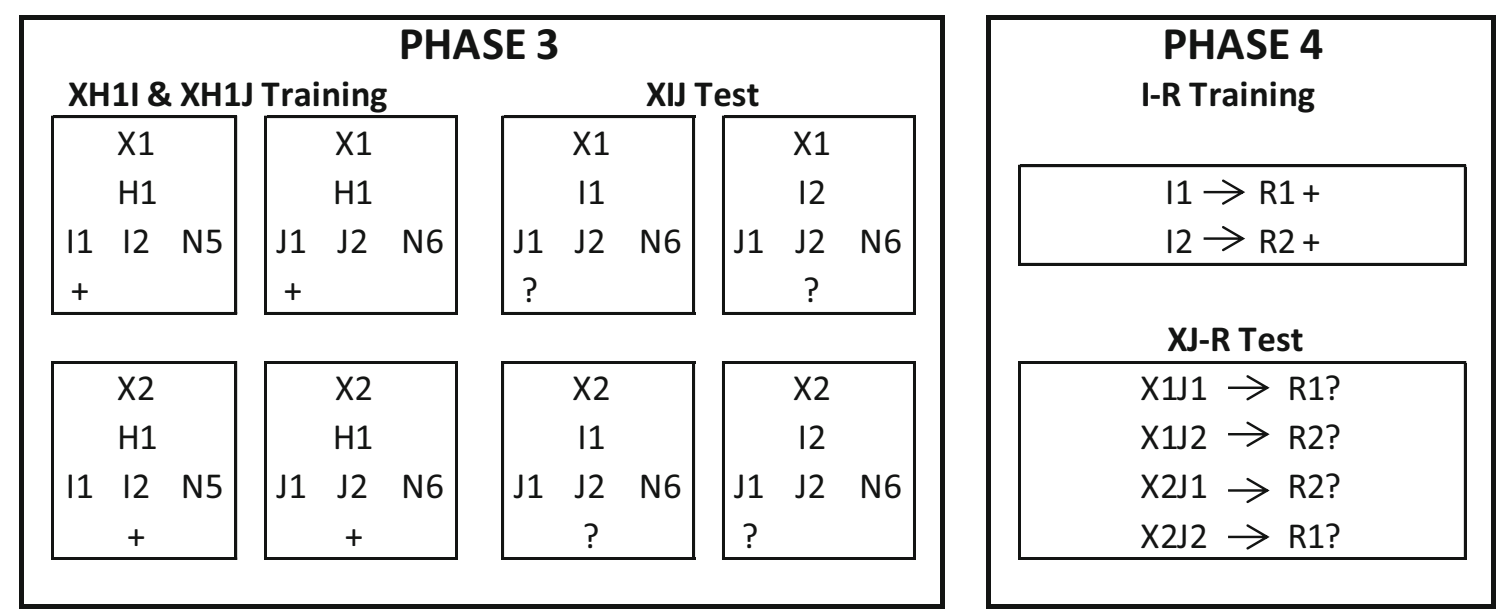

Fig. 5 Discriminations trained and tested in Phases 3 and 4. In the diagram for Phase 3, each inner panel corresponds to a trial type of a discrimination. Contextual cues are represented at the top of each panel, samples in the middle, and comparisons at the bottom. In Phase 4, each inner panel corresponds to a discrimination. Antecedent stimuli are

N3 was never correct). In EG discrimination, the relations trained were E1G1 and E2G2 (choosing N4 was never correct). EF and EG established the prerequisites for the formation of the E1F1G1 and E2F2G2 classes. Thus, in the XFG test, the correct relations were F1G1 and F2G2 in the presence of $\mathrm{X} 1$ (the supposed cue for equivalence), and F1G2 and F2G1 in the presence of $\mathrm{X} 2$ (the supposed cue for nonequivalence). The FG and GF tests were included to confirm that the training of EF and EG generated the E1F1G1 and E2F2G2 classes. Thus, the correct relations in the FG test were F1G1 and F2G2, and in the GF test were G1F1 and G2F2.

The sequence of blocks presented in Phase 2, the discriminations trained and tested in each block, and the number of trials per block appear in Tables 1, 2 and 3. EF and EG were trained separately in Blocks 1 and 2, and then intermixed in Block 3, for facilitating their acquisition and maintenance. When participants achieved the criterion for passing the XFG test (12 correct out of 12 trials), they moved on to Block 5; otherwise, they moved back to Block 1 (we had scheduled to send back a participant to Block 1 a maximum of three times). The FG and GF tests from Blocks 5 and 6 were presented only once, because they were intended to evaluate whether the E1F1G1 and E2F2G2 classes had been previously established. Thus, participants moved on to Phase 3 regardless of their results in Blocks 5 and 6. Nevertheless, we established a criterion for passing these tests of 11 correct responses out of 12 trials (we allowed one error as Blocks 5 and 6 were presented only once).

Phase 3: $X 1$ and $X 2$ in combinatorial entailment tests. In Phase 3, we evaluated whether X1 and X2 displayed the same functions as SAME and OPPOSITE in supposed tests for the combinatorial entailment of the relations of sameness of opposition in RFT studies. We trained and tested for shown to the left of the arrow and correct responses in the presence of such stimuli are shown to the right. Correct choices for each trial are indicated by a plus symbol, for correct choices followed by consequences, or by a quotation mark, for correct choices not followed by consequences

discriminations analogous to those form RFT studies on sameness and opposition, with the exception that SAME was replaced by X1, and OPPOSITE was replaced by X2.

Phase 3 consisted of training the XH1I and XH1J discriminations, followed by the XIJ test (see Fig. 5, left panel). In the XH1I discrimination, I1 was the correct choice in the presence of X1 and H1, and I2 was the correct choice in the presence of $\mathrm{X} 2$ and $\mathrm{H} 1$ (selecting N5 was never correct). In the XH1J discrimination, $\mathrm{J} 1$ was the correct choice in the presence of $\mathrm{X} 1$ and $\mathrm{H} 1$, and $\mathrm{J} 2$ was the correct choice in the presence of $\mathrm{X} 2$ and H1 (selecting N6 was never correct). Notice that the sample presented across all trials from XH1I and XH1J was always the same, H1, as in RFT studies where typical training trials are SAME-A1B1, OPPOSITE-A1B2, SAME-A1C1, and OPPOSITE-A1C2. Consistently with the outcomes found in RFT studies (see Fig. 2, left panel), in the XIJ test we defined $\mathrm{J} 1$ as the correct choice in the presence of $\mathrm{X} 1$ and $\mathrm{I} 1, \mathrm{~J} 2$ as the correct choice in the presence of X1 and I2, J2 as the correct choice in the presence of X2 and $\mathrm{I} 1$, and $\mathrm{J} 2$ as the correct choice in the presence of X2 and I1.

The sequence of blocks presented in Phase 3, the discriminations trained or tested in each block, and the number of trials per block appear in Tables 1, 2 and 3. XH1I and XH1J were trained separately in Blocks 1 and 2, and intermixed in Block 3, for facilitating their acquisition and maintenance. When participants achieved the criterion for passing the XIJ test (12 correct responses out of 12 trials), they moved on to Phase 4; otherwise, they moved back to Block 1. We had scheduled to repeat this sequence a maximum of four times.

Phase 4: $\mathrm{X} 1$ and $\mathrm{X} 2$ in transformation of function tests. In Phase 4, we evaluated whether X1 and X2 displayed the same functions as SAME and OPPOSITE in RFT tests that supposedly measured the transformation of functions in accordance 


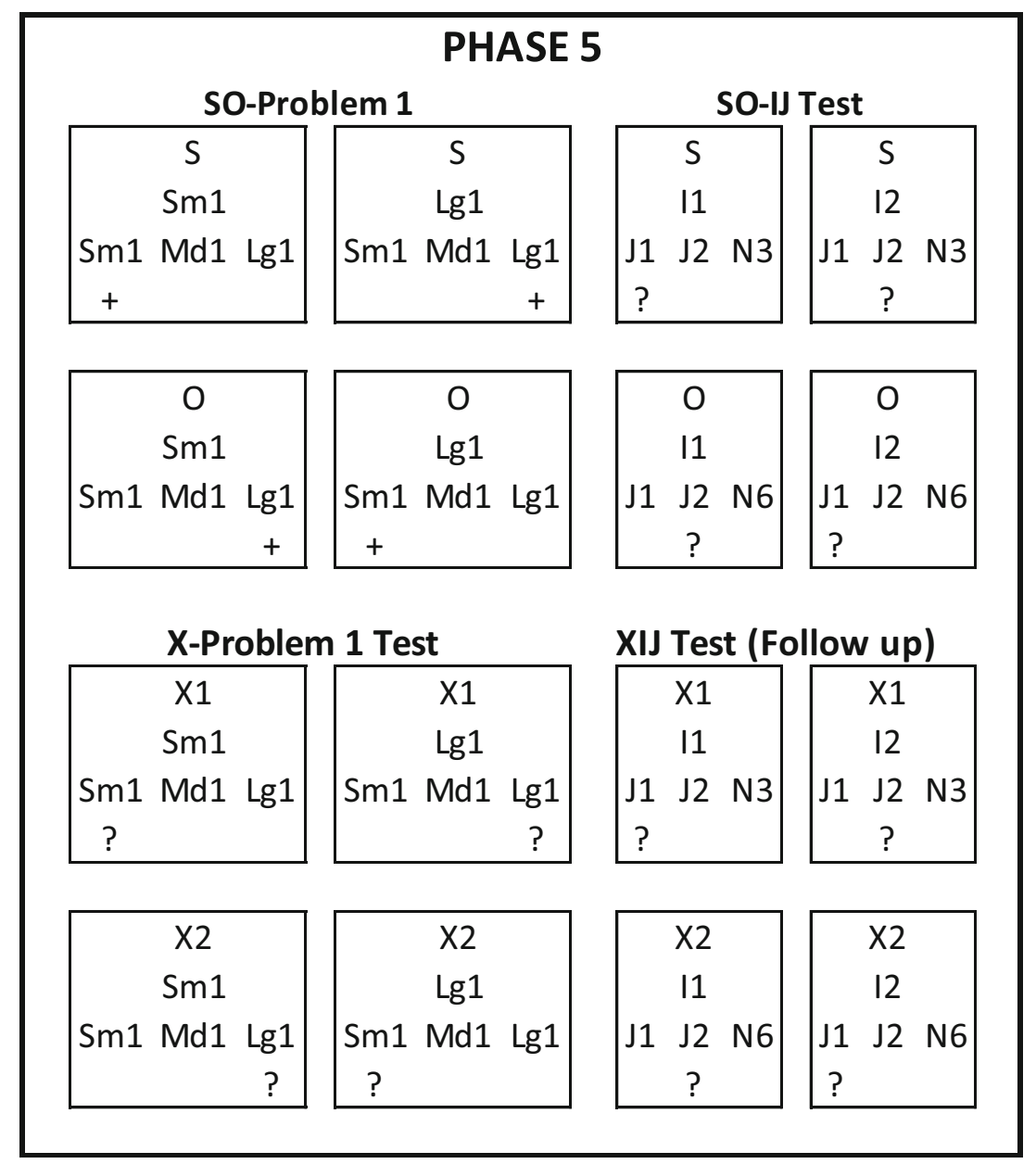

Fig. 6 Some of the discriminations trained and tested in Phase 5. Each inner panel corresponds to a trial type from a discrimination. Contextual cues are represented at the top of each panel, samples in the middle, and comparisons at the bottom. Correct choices for each trial are indicated by a plus symbol, for correct choices followed by consequences, or by a quotation mark, for correct choices not followed by consequences with sameness of opposition (i.e., how the functions of stimuli included in frames of sameness and opposition changed or were transformed by the presence of contextual cues for sameness and opposition; e.g., Hayes et al., 2001). As in Phase 3, in Phase 4 we trained and tested for discriminations analogous to those from RFT studies on sameness and opposition, with the exception that SAME was replaced by $\mathrm{X} 1$, and OPPOSITE was replaced by $\mathrm{X} 2$. In particular, we trained and tested simple discriminations analogous to discriminations trained and tested by Roche et al. (2000, Experiment 1).

Phase 4 consisted of training the I-R simple discrimination, followed by the XJ-R test (see Fig. 5, right panel). In the I-R discrimination, I1 and I2 were presented successively as discriminative stimuli. In the presence of I1, the correct response was pressing the space bar of the computer's keyboard twice (denominated R1), while in the presence of I2, the correct response was pressing the space bar five times (denominated R2).

In the XJ-R test, we presented $\mathrm{X} 1$ and $\mathrm{X} 2$ as contextual cues together with the discriminative stimuli J1 and J2. The correct responses in the XJ-R test were defined assuming that $\mathrm{X} 1$ and $\mathrm{X} 2$ were functionally equivalent to the contextual cues SAME and OPPOSITE in previous studies (e.g., Roche et al., 2000). Thus, the correct responses in XJ-R test were pressing the space bar twice in the presence of X1 and $\mathrm{J} 1$ and in the presence of $\mathrm{X} 2$ and $\mathrm{J} 2$; and pressing the space bar five times in the presence of $\mathrm{X} 2$ and $\mathrm{J} 1$ in the presence of $\mathrm{X} 1$ and $\mathrm{J} 2$.

The sequence of blocks presented in Phase 4, the discriminations trained or tested in each block, and the number of trials per block appear in Tables 1,2 and 3. This trainingtesting sequence was presented only once. Block 2 was included to assess whether participants could demonstrate I-R in the absence of differential consequences. We adopted a criterion for passing the XJR test of least seven correct responses out of eight trials.

Phase 5: $X 1$ and $X 2$ as functionally equivalent to SAME and OPPOSITE. See Fig. 6. In Phase 5, we evaluated whether X1 and X2 were functionally equivalent to SAME and OPPOSITE, in congruence with our hypothesis that the 
SAME and OPPOSITE cues from RFT studies were cues for equivalence and nonequivalence, and thus they should be functionally interchangeable with X1 and X2 (see Fig. 6). The sequence of blocks presented in Phase 5, the discriminations trained or tested in each block, and the number of trials per block appear in Tables 1, 2 and 3.

In Blocks 1-3, participants were presented with three NARR problems in which they were trained to select comparisons physically identical to the samples in the presence of SAME, and comparisons the most physically dissimilar to the samples in the presence of OPPOSITE (the type of NARR used in RFT studies). These problems were labeled SO-Problems ("S" stands for SAME and "O" for OPPOSITE). In Block 1, we trained the SO-Problem 1 where Sm1 and Lg1 were the samples, and Sm1, Md1, and Lg1 were the comparisons. In Block 2, we trained the SO-Problem 2 where Sm2 and Lg2 were the samples, and $\mathrm{Sm} 2, \mathrm{Md} 2$, and Lg2 were the comparisons. In Block 3, we trained the SOProblem 3 where Sm3 and Lg3 were the samples, and Sm3, $\mathrm{Md} 3$, and $\operatorname{Lg} 3$ were the comparisons.

In Block 4, participants were presented with a test including new NARR problems to evaluate whether SAME controlled the selection of comparisons identical to the samples, and OPPOSITE controlled the selection of comparisons the most dissimilar to the samples, with stimulus sets not used in training. In the SO-Problem 4, Sm4 and Lg4 were the samples and $\mathrm{Sm} 4, \mathrm{Md} 4$, and $\mathrm{Lg} 4$ were the comparisons. In the SO-Problem 5, Sm5 and Lg5 were the samples and $\mathrm{Sm} 5, \mathrm{Md} 5$, and Lg5 were the comparisons. In the SO-Problem 6, where Sm6 and Lg6 were the samples, and Sm6, Md6, and Lg6 were the comparisons. The criterion for passing the tests of Block 4 was 12 correct responses out of 12 trials. The training-testing sequence of Blocks 1-4 was presented only once. We did not allow any errors in Block 4 as learning the functions of SAME and OPPOSITE was required for passing subsequent tests.

In Block 5, participants were presented with a tests including the same problems as Blocks 1-3, except that SAME and OPPOSITE were replaced by X1 and X2. We expected that $\mathrm{X} 1$ would control the selection of comparisons identical to the samples and X2 would control comparisons the most dissimilar to the samples (i.e., we expected X1 to be equivalent to SAME and X2 to be equivalent to OPPOSITE).

In Block 6, participants were presented with a test identical to XIJ test from Phase 3, except that X1 and X2 were replace by SAME and OPPOSITE. This test was labeled SO-IJ. We expected that SAME would control the selection of $\mathrm{J} 1$ in the presence of $\mathrm{I} 1$, and $\mathrm{J} 2$ in the presence of $\mathrm{I} 2$ (i.e., we expected that SAME would be equivalent to X1). We also expected that participants would control the selection of $\mathrm{J} 2$ in the presence of $\mathrm{I} 1$, and the selection of $\mathrm{J} 1$ in the presence of $\mathrm{I} 2$ (i.e., we expected that OPPOSITE would be equivalent to $\mathrm{X} 2$ ).
In Block 7, participants were presented again with the XIJ test from Phase 3. It was presented to evaluate whether participants maintained this discrimination, and to discard that potential failures in the SO-IJ test could be attributed to the "forgetting" of the relations between the I and J stimuli. The test sequence of Blocks 5-7 was presented once, and the experiment ended after the presentation of Block 7 . The criterion for passing each test was at least 11 correct responses out of 12 trials.

\section{Results}

The results of the three participants are shown in Tables 1, 2 and 3. In Phase 1, the three participants completed two (P102 and P103) and three (P101) training-testing cycles. They achieved a minimum of $95 \%$ correct responses in the unprompted trials of Blocks 1-7 of each cycle. They responded correctly to most trials of each XCD test and achieved the criterion for the XCD test (12 correct responses out of 12 trials).

In Phase 2, the three participants completed one (P101 and P103) and two (P102) training-testing cycles with a minimum of $79 \%$ correct responses in the Blocks $1-3$ of each cycle. Finally, they achieved the criterion for the XFG test (12 correct responses out of 12 trials) and for the FG and GF tests (at least 11 out of 12 correct responses).

In Phase 3, the three participants completed one (P103), two (P102) and four (P101) training-testing cycles with a minimum of $86 \%$ correct responses in the Blocks 1-3 of each cycle. They responded correctly to at least 10 out of the 12 trials of each XIJ test, with the exception of P101 in his second cycle where he responded correctly to eight trials, and they achieved the criterion for the XIJ test (12 correct response out of 12 trials).

In Phase 4, the three participants achieved $100 \%$ correct responses in Blocks 1 and 2. In the XJ-R test, P101 responded correctly to seven trials, while $\mathrm{P} 102$ and $\mathrm{P} 103$ responded correctly to eight trials. Thus, all three participants achieved the criterion for the XJ-R test.

In Phase 5, the three participants obtained 84\%, 66\%, and $100 \%$ correct responses in unprompted trials of Blocks 1-3. They also reached the criterion for the for the SO-Problems 4 6 tests (12 correct responses out of 12 trials), the X-Problems 1-3 tests, the SO-IJ test and XIJ test (at least 11 correct responses out of 12 trials).

\section{Discussion}

In Phase 1, three college students were trained to maintain previously acquired conditional discriminations in the presence of $\mathrm{X} 1$, and to reverse those discriminations in the 
Table 1 Results of Participant P101

\begin{tabular}{|c|c|c|c|c|c|c|c|}
\hline Block & Discrimination & Block type & $\begin{array}{l}\text { Trials per } \\
\text { block }\end{array}$ & Results & & & \\
\hline \multicolumn{8}{|l|}{ Phase 1} \\
\hline Bq. 1 & A1B1 & Training & $3 p+3$ & $6 / 6$ & $6 / 6$ & $6 / 6$ & \\
\hline Bq. 2 & $\mathrm{~A} 2 \mathrm{~B} 2$ & Training & $3 p+3$ & $6 / 6$ & $6 / 6$ & $6 / 6$ & \\
\hline Bq. 3 & $\mathrm{AB}$ & Training & 12 & $12 / 12$ & $12 / 12$ & $12 / 12$ & \\
\hline Bq. 4 & $\mathrm{X} 1 \mathrm{AB}$ & Training & 12 & $12 / 12$ & $12 / 12$ & $12 / 12$ & \\
\hline Bq. 5 & $\mathrm{X} 2 \mathrm{AB}$ & Training & 12 & $20 / 22$ & $12 / 12$ & $12 / 12$ & \\
\hline Bq. 6 & $\mathrm{XAB}$ & Training & 12 & $12 / 12$ & $17 / 18$ & $13 / 14$ & \\
\hline Bq. 7 & $\mathrm{CD}$ & Training & 12 & $25 / 28$ & $12 / 12$ & $12 / 12$ & \\
\hline Bq. 8 & $\mathrm{XCD}$ & Test & 12 & $11 / 12$ & $10 / 12$ & $12 / 12$ & \\
\hline \multicolumn{8}{|l|}{ Phase 2} \\
\hline Bq. 1 & $\mathrm{EF}$ & Training & 12 & $14 / 18$ & & & \\
\hline Bq. 2 & EG & Training & 12 & $13 / 16$ & & & \\
\hline Bq. 3 & EF EG & Training & 12 & $12 / 12$ & & & \\
\hline Bq. 4 & XFG & Test & 12 & $12 / 12$ & & & \\
\hline Bq. 5 & FG & Test & 12 & $12 / 12$ & & & \\
\hline Bq. 6 & GF & Test & 12 & $12 / 12$ & & & \\
\hline \multicolumn{8}{|l|}{ Phase 3} \\
\hline Bq. 1 & XH1I & Training & 12 & $13 / 17$ & $12 / 12$ & $12 / 12$ & $12 / 12$ \\
\hline Bq. 2 & XH1J & Training & 12 & $12 / 12$ & $44 / 50$ & $12 / 12$ & $12 / 13$ \\
\hline Bq. 3 & XH1I XH1J & Training & 12 & $15 / 17$ & $12 / 12$ & $13 / 14$ & $39 / 43$ \\
\hline Bq. 4 & XIJ & Test & 12 & $10 / 12$ & $8 / 12$ & $11 / 12$ & $12 / 12$ \\
\hline \multicolumn{8}{|l|}{ Phase 4} \\
\hline Bq. 1 & I-R & Training & $6 p+6$ & $12 / 12$ & & & \\
\hline Bq. 2 & I-R & Test & 6 & $6 / 6$ & & & \\
\hline Bq. 3 & XJ-R & Test & 8 & $7 / 8$ & & & \\
\hline \multicolumn{8}{|l|}{ Phase 5} \\
\hline Bq. 1 & SO-Problem 1 & Training & $3 p+4$ & $8 / 10$ & & & \\
\hline Bq. 2 & SO-Problem 2 & Training & $3 p+4$ & $7 / 7$ & & & \\
\hline Bq. 3 & SO-Problem 3 & Training & $3 p+4$ & $10 / 11$ & & & \\
\hline Bq. 4 & SO-Problems 4-6 & Test & 12 & $12 / 12$ & & & \\
\hline Bq. 5 & X-Problems 1-3 & Test & 12 & $11 / 12$ & & & \\
\hline Bq. 6 & SO-IJ & Test & 12 & $12 / 12$ & & & \\
\hline Bq. 7 & XIJ & Test & 12 & $12 / 12$ & & & \\
\hline
\end{tabular}

Note. The table columns show the presentation order of the blocks from each phase, the discriminations presented in each block, the block type (training or test blocks), the number of trials programmed for each block, and the results of Participant P101 (correct responses/total trials). The expression "Bq." stands for block; " $3 \mathrm{p}$ " and " $6 \mathrm{p}$ " stand for 3 and 6 prompted trials, respectively

presence of X2. The tests presented in Phase 1 demonstrated that $\mathrm{X} 1$ and $\mathrm{X} 2$ became generalized contextual cues for maintaining and reversing new conditional discriminations. These results replicated those of previous studies (Pérez-González et al., 2014; Pérez-González et al., 2015; Pérez-González \& Martínez, 2007; Pérez-González \& Serna, 2003; Serna \& Pérez-González, 2003). The tests presented in Phase 2 suggest that the training provided in Phase 1 also made of X1 and X2 cues for equivalence and nonequivalence. The tests presented in Phases 3 and 4 showed that X1 and X2 controlled the same types of derived responding as supposed cues for sameness and opposition in RFT studies. Specifically, these tests showed that X1 and X2 controlled the same types of derived responding as SAME and OPPOSITE in tests that supposedly evaluated the combinatorial entailment of the frames of sameness and opposition, and the transformation of function in accordance with those frames. The tests presented in Phase 5 confirmed the functional equivalence of X1 and SAME and $\mathrm{X} 2$ and OPPOSITE.

The functional equivalence of X1 and SAME was expected because both cues were supposed to function as cues for SE or the frame as sameness, as SE is referred to in RFT. The 
Table 2 Results of Participant P102 (see note for Table 1)

\begin{tabular}{|c|c|c|c|c|c|}
\hline Block & Discrimination & Block type & $\begin{array}{l}\text { Trials per } \\
\text { block }\end{array}$ & Results & \\
\hline \multicolumn{6}{|l|}{ Phase 1} \\
\hline Bq. 1 & A1B1 & Training & $3 p+3$ & $6 / 6$ & \\
\hline Bq. 2 & $\mathrm{~A} 2 \mathrm{~B} 2$ & Training & $3 p+3$ & $6 / 6$ & \\
\hline Bq. 3 & $\mathrm{AB}$ & Training & 12 & $12 / 12$ & $31 / 33$ \\
\hline Bq. 4 & $\mathrm{X} 1 \mathrm{AB}$ & Training & 12 & $12 / 12$ & $12 / 13$ \\
\hline Bq. 5 & $\mathrm{X} 2 \mathrm{AB}$ & Training & 12 & $12 / 15$ & $27 / 29$ \\
\hline Bq. 6 & $\mathrm{XAB}$ & Training & 12 & $35 / 52$ & $12 / 12$ \\
\hline Bq. 7 & $\mathrm{CD}$ & Training & 12 & $17 / 24$ & $12 / 12$ \\
\hline Bq. 8 & $\mathrm{XCD}$ & Test & 12 & $6 / 12$ & $12 / 12$ \\
\hline \multicolumn{6}{|l|}{ Phase 2} \\
\hline Bq. 1 & $\mathrm{EF}$ & Training & 12 & $20 / 29$ & $12 / 12$ \\
\hline Bq. 2 & EG & Training & 12 & $16 / 20$ & $12 / 12$ \\
\hline Bq. 3 & EF EG & Training & 12 & $12 / 12$ & $12 / 12$ \\
\hline Bq. 4 & XFG & Test & 12 & $11 / 12$ & $12 / 12$ \\
\hline Bq. 5 & FG & Test & 12 & & $12 / 12$ \\
\hline Bq. 6 & GF & Test & 12 & & $12 / 12$ \\
\hline \multicolumn{6}{|l|}{ Phase 3} \\
\hline Bq. 1 & XH1I & Training & 12 & $22 / 24$ & $12 / 12$ \\
\hline Bq. 2 & XH1J & Training & 12 & $13 / 18$ & $32 / 34$ \\
\hline Bq. 3 & XH1I XH1J & Training & 12 & $12 / 12$ & $12 / 12$ \\
\hline Bq. 4 & XIJ & Test & 12 & $11 / 12$ & $12 / 12$ \\
\hline \multicolumn{6}{|l|}{ Phase 4} \\
\hline Bq. 1 & I-R & Training & $6 p+6$ & $12 / 12$ & \\
\hline Bq. 2 & I-R & Test & 6 & $6 / 6$ & \\
\hline Bq. 3 & XJ-R & Test & 8 & $8 / 8$ & \\
\hline \multicolumn{6}{|l|}{ Phase 5} \\
\hline Bq. 1 & SO-Problem 1 & Training & $3 p+4$ & $9 / 15$ & \\
\hline Bq. 2 & SO-Problem 2 & Training & $3 p+4$ & $7 / 7$ & \\
\hline Bq. 3 & SO-Problem 3 & Training & $3 p+4$ & $7 / 9$ & \\
\hline Bq. 4 & SO-Problems 4-6 & Test & 12 & $12 / 12$ & \\
\hline Bq. 5 & X-Problems 1-3 & Test & 12 & $12 / 12$ & \\
\hline Bq. 6 & SO-IJ & Test & 12 & $12 / 12$ & \\
\hline Bq. 7 & XIJ & Test & 12 & $12 / 12$ & \\
\hline
\end{tabular}

functional equivalence of $\mathrm{X} 2$ and OPPOSITE is more relevant for the purpose of this research. It suggests that OPPOSITE functioned as cue for nonequivalence in RFT studies, rather than as cue for the frame of opposition. Notice that the tests presented in Phase 2 suggest that X2 became a cue for nonequivalence as a result of the training provided in Phase 1. Therefore, X2 probably functioned as a cue for nonequivalence in the tests presented in Phases 3 and 4, which were analogous to the combinatorial entailment tests and the transformation of function tests from RFT studies. In addition, OPPOSITE could have also functioned as a cue for nonequivalence in RFT studies because it was pretrained as a cue for excluding the selection of comparisons identical (i.e., equivalent) to the samples (e.g., Steele \& Hayes, 1991). Therefore, a possible explanation of the functional equivalence of X2 and OPPOSITE is that both functioned as cues for nonequivalence.

An alternative explanation of the functional equivalence of $\mathrm{X} 2$ and OPPOSITE would be that these cues were equivalent because both were cues for the frame of opposition. X2 was not trained as a cue for selecting comparisons the most dissimilar to the samples, the training that according to RFT transforms an arbitrary stimulus into a cue for the frame of opposition. Thus, it seems difficult to explain why X2 would have become a cue for the frame of opposition and not for any other frame. It remains possible that reversing the initially 
Table 3 Results of Participant P103 (see note of Table 1)

\begin{tabular}{|c|c|c|c|c|c|}
\hline Block & Discrimination & Block type & $\begin{array}{l}\text { Trials per } \\
\text { block }\end{array}$ & Results & \\
\hline \multicolumn{6}{|l|}{ Phase 1} \\
\hline Bq. 1 & A1B1 & Training & $3 p+3$ & $6 / 6$ & \\
\hline Bq. 2 & $\mathrm{~A} 2 \mathrm{~B} 2$ & Training & $3 p+3$ & $6 / 6$ & \\
\hline Bq. 3 & $\mathrm{AB}$ & Training & 12 & $12 / 12$ & $12 / 12$ \\
\hline Bq. 4 & $\mathrm{X} 1 \mathrm{AB}$ & Training & 12 & $12 / 13$ & $12 / 12$ \\
\hline Bq. 5 & $\mathrm{X} 2 \mathrm{AB}$ & Training & 12 & $12 / 13$ & $15 / 16$ \\
\hline Bq. 6 & XAB & Training & 12 & $16 / 17$ & $12 / 12$ \\
\hline Bq. 7 & $\mathrm{CD}$ & Training & 12 & $13 / 15$ & $12 / 12$ \\
\hline Bq. 8 & $\mathrm{XCD}$ & Test & 12 & $11 / 12$ & $12 / 12$ \\
\hline \multicolumn{6}{|l|}{ Phase 2} \\
\hline Bq. 1 & $\mathrm{EF}$ & Training & 12 & $12 / 13$ & \\
\hline Bq. 2 & EG & Training & 12 & $12 / 14$ & \\
\hline Bq. 3 & EF EG & Training & 12 & $12 / 12$ & \\
\hline Bq. 4 & XFG & Test & 12 & $12 / 12$ & \\
\hline Bq. 5 & FG & Test & 12 & $12 / 12$ & \\
\hline Bq. 6 & GF & Test & 12 & $12 / 12$ & \\
\hline \multicolumn{6}{|l|}{ Phase 3} \\
\hline Bq. 1 & XH1I & Training & 12 & $12 / 16$ & \\
\hline Bq. 2 & XH1J & Training & 12 & $13 / 15$ & \\
\hline Bq. 3 & XH1I XH1J & Training & 12 & $12 / 12$ & \\
\hline Bq. 4 & XIJ & Test & 12 & $12 / 12$ & \\
\hline \multicolumn{6}{|l|}{ Phase 4} \\
\hline Bq. 1 & I-R & Training & $6 p+6$ & $12 / 12$ & \\
\hline Bq. 2 & I-R & Test & 6 & $6 / 6$ & \\
\hline Bq. 3 & XJ-R & Test & 8 & $8 / 8$ & \\
\hline \multicolumn{6}{|l|}{ Phase 5} \\
\hline Bq. 1 & SO-Problem 1 & Training & $3 p+4$ & $7 / 7$ & \\
\hline Bq. 2 & SO-Problem 2 & Training & $3 p+4$ & $7 / 7$ & \\
\hline Bq. 3 & SO-Problem 3 & Training & $3 p+4$ & $7 / 7$ & \\
\hline Bq. 4 & SO-Problems 4-6 & Test & 12 & $12 / 12$ & \\
\hline Bq. 5 & X-Problems 1-3 & Test & 12 & $12 / 12$ & \\
\hline Bq. 6 & SO-IJ & Test & 12 & $12 / 12$ & \\
\hline Bq. 7 & XIJ & Test & 12 & $12 / 12$ & \\
\hline
\end{tabular}

trained relations in X2 yielded opposition - it would just illustrate a new way to do it. In any case, what is interesting is that the standard RFT demonstrations claimed to support opposition may be interpreted in other terms.

Steele and Hayes (1991) admitted that apparent AARR in accordance with sameness and opposition could be alternatively explained by contextual control over equivalence and nonequivalence. Later on, RFT researchers dismissed this alternative explanation. The apparent reason for it was methodological. Steele and Hayes' presented two comparisons per trial. Therefore, an explanation of their results in terms of contextual control over equivalence and nonequivalence was straightforward. For instance, in the presence of SAME and
$\mathrm{B} 2$, the comparisons were $\mathrm{C} 1$ and $\mathrm{C} 2$. Participants selected $\mathrm{C} 2$. This outcome can be explained by the exclusion of $\mathrm{C} 1$, a comparison nonequivalent to $\mathrm{B} 2$, in the presence of a cue for equivalence, SAME. As the only alternative to $\mathrm{C} 1$ was $\mathrm{C} 2$, participants selected C2. In studies that came after Steele and Hayes (1991), participants were presented with three comparisons per trial. For instance, with SAME and B2, the comparisons were $\mathrm{C} 1, \mathrm{C} 2$ and one or several $\mathrm{N}$ comparisons, which were never designated as correct choices during training. Participants in these studies typically selected C2. This outcome apparently rules out the explanation based on contextual control over equivalence and nonequivalence, because one may think that had participants responded by the exclusion 
of $\mathrm{C} 1$ in the presence of B2 and SAME, then they should have selected any of the alternatives to $\mathrm{C} 1$ (i.e., $\mathrm{C} 2$ or the $\mathrm{N}$ comparisons) with equal probability. For RFT researchers, the selection of $\mathrm{C} 2$ in the presence of B2 and SAME can only be explained by the existence of a relation of sameness or equivalence between B2 and C2. That relation would have been derived via combinatorial entailment from the opposite relation between $\mathrm{A} 1$ and $\mathrm{B} 2$, and $\mathrm{A} 1$ and $\mathrm{C} 2$. RFT predicts that if $\mathrm{B} 2$ is the opposite of $\mathrm{A} 1$, and $\mathrm{C} 2$ is the opposite of $\mathrm{A} 1$, then $\mathrm{B} 2$ and $\mathrm{C} 2$ will be the same or equivalent. Thus, the selection of $\mathrm{C} 2$ in the presence of $\mathrm{B} 2$ and the cue for equivalence (SAME) would be in accordance with RFT predictions and could not be explained by contextual control over equivalence and nonequivalence. This explanation seems week because the selection of $\mathrm{C} 2$ in the presence of B2 and SAME can still be explained by contextual control over equivalence and nonequivalence: Participants may have selected $\mathrm{C} 2$ in the presence of B2 and SAME because of the exclusion of $\mathrm{C} 1$ - the comparison nonequivalent to $\mathrm{C} 2$ - and the exclusion of the $\mathrm{N}$ comparisons. Notice that the $\mathrm{N}$ comparisons were never designated as correct during training. Thus, participants may have excluded their selection in all test trials for that reason.

The results of this research strength the argument that apparent AARR in accordance with sameness and opposition can be explained by contextual control over equivalence and nonequivalence, even when three comparisons per trials are presented. The participants in the present study selected J2 in the presence of $\mathrm{I} 2$ and $\mathrm{X} 1$, a selection that is analogous to selecting $\mathrm{C} 2$ in the presence of B2 and SAME. An explanation of this outcome in terms of sameness and opposition would require that $\mathrm{X} 2$ had functioned as a cue for opposition, and therefore the existence of opposition relations between I2 and $\mathrm{H} 1$, and $\mathrm{J} 2$ and $\mathrm{H} 1$, and of a derived relation of sameness or equivalence relation between $\mathrm{I} 2$ and $\mathrm{J} 2$. The hypothesis that $\mathrm{X} 2$ functioned as a cue for the frame of opposition cannot be ruled out (see above). This hypothesis, however, seems much weaker than the hypothesis that OPPOSITE functioned as a cue for the frame of opposition RFT studies. OPPOSITE was pretrained as a cue for selecting comparisons the most physically dissimilar to the samples, whereas X2 was not. The alternative hypothesis that X2 functioned as a cue for nonequivalence seems at least as plausible. In summary, contrary to their claims, RFT researchers did not provide any conclusive demonstration of AARR in accordance with sameness and opposition, and the apparent demonstration of this process can be alternatively explained by contextual control over equivalence and nonequivalence.

The demonstration that apparent AARR in accordance with the frames of sameness and opposition can be explained by contextual control over equivalence and nonequivalence is relevant for the debate about the origin of derived stimulus relations (e. g., Horne \& Lowe, 1996; Hayes et al., 2001;
Sidman, 2000; Rehfeldt \& Hayes, 1998; Tonneau, 2001). RFT researchers claimed that their theory has a comparative advantage over alternative theories exclusively focused on SE because RFT would be the only account that can explain the origin of derived stimulus relations other than equivalence (e.g., Roche et al., 2000). The results of this research challenge such claims, at least in regard to the frames of sameness and opposition. Actually, this research supports the conclusion that apparent AARR in accordance with sameness and opposition can be explained by all theories about the origin of SE. To do so, such theories have only to appeal to contextual control over conditional discrimination performance and responding by exclusion, in addition to the mechanisms that they propose to explain the origin of SE. This challenge to RFT claims seems especially significant given that the frames of sameness and opposition are among the frames more frequently addressed in RFT literature (e.g., Dymond, May, Munnelly, \& Hoon, 2010).

It is still to be seen whether contextual control over equivalence and nonequivalence can account for apparent AARR in accordance with other relational frames addressed in RFT research, such as the frames of distinction, comparison, or hierarchical frames (e.g., Hayes et al., 2001). The outcomes of the present research call for more research investigating this possibility. In regard to the frames of sameness and opposition, this study was conducted with only three participants. Although this number is frequent in studies of this type and the careful procedure brought very consistent results, it would benefit from additional replications. Of special interest will be extension of the present study with procedures that allow results that could be interpreted either by contextual control over equivalence and exclusion or by RFT theory.

\section{References}

Bennett, M., Hermans, D., Dymond, S., Vervoort, E., \& Baeyens, F. (2015). From bad to worse: Symbolic equivalence and opposition in fear generalization. Cognition and Emotion, 29, 1137-1145.

Cassidy, S., Roche, B., \& Hayes, S. C. (2011). A relational frame training intervention to raise intelligence quotients: A pilot study. The Psychological Record, 61, 173-198.

Clayton, M. C., \& Hayes, L. J. (1999). Conceptual differences in the analysis of stimulus equivalence. The Psychological Record, 49, $145-161$

de Rose, J. C., de Souza, D. G., \& Hanna, E. S. (1996). Teaching reading and spelling: Exclusion and stimulus equivalence. Journal of Applied Behavior Analysis, 29, 451-469.

Dymond, S., May, R. J., Munnelly, A., \& Hoon, A. E. (2010). Evaluating the evidence base for Relational Frame Theory: A citation analysis. The Behavior Analyst, 33, 97-117.

Dymond, S., Roche, B., Forsyth, J. P., Whelan, R., \& Rhoden, J. (2007). Transformation of avoidance response functions in accordance with same and opposite relational frames. Journal of the Experimental Analysis of Behavior, 88, 249-262. 
Dymond, S., Roche, B., Forsyth, J. P., Whelan, R., \& Rhoden, J. (2008). Derived avoidance learning: Transformation of avoidance response functions in accordance with same and opposite relational frames. The Psychological Record, 58, 269-286.

Dymond, S., Tsz, C. N., \& Whelan, R. (2013). Establishing arbitrarily applicable relations of same and opposite with the relational completion procedure: Selection-based feedback. The Psychological Record, 63, 111-130.

Dymond, S., \& Whelan, R. (2010). Derived relational responding: A comparison of match-to-sample and the relational completion procedure. Journal of the Experimental Analysis of Behavior, 94, 3755.

Hayes, S. C., Barnes-Holmes, D., \& Roche, B. (Eds.). (2001). Relational frame theory: A post-Skinnerian account of human language and cognition. New York, NY: Plenum Press.

Horne, P. J., \& Lowe, C. F. (1996). On the origins of naming and other symbolic behavior. Journal of the Experimental Analysis of Behavior, 65, 185-241.

Lynch, D. C., \& Green, G. (1991). Development and cross-modal transfer of contextual control of emergent stimulus relations. Journal of the Experimental Analysis of Behavior, 56, 139-154.

Ninness, C., Dixon, M., Barnes-Holmes, D., Rehfeldt, R. A., Rumph, R., McCuller, G., \& McGinty, J. (2009). Constructing and deriving reciprocal trigonometric relations: a functional analytic approach. Journal of Applied Behavior Analysis, 42, 191-208.

Perez, W. F., de Almeida, J. H., \& de Rose, J. C. (2015). Transformation of meaning through relations of sameness and opposition. The Psychological Record, 65, 679-689.

Pérez-González, L. A., Álvarez, E., Calleja, A., \& Fernández, A. (2014). Transfer of three functions of contextual cues in conditional discriminations. The Psychological Record, 65, 277-287.

Pérez-González, L. A., Díaz, E., Fernández-García, S., \& Baizán, C. (2015). Stimuli with identical contextual functions taught independently become functionally equivalent. Learning and Behavior, 43, $113-128$.

Pérez-González, L. A., \& Martínez, H. (2007). Control by contextual cues in novel second-order conditional discriminations. The Psychological Record, 57, 117-143.

Pérez-González, L. A., \& Serna, R. W. (2003). Transfer of specific contextual functions to novel conditional discriminations. Journal of the Experimental Analysis of Behavior, 79, 395-408.
Rehfeldt, R. A., \& Hayes, L. J. (1998). The operant-respondent distinction revisited: Towards an understanding of stimulus equivalence. The Psychological Record, 48, 187-210.

Roche, B., \& Barnes, D. (1996). Arbitrarily applicable relational responding and human sexual categorization: A critical test of the derived difference relation. The Psychological Record, 46, 451-475.

Roche, B., \& Barnes, D. (1997). A transformation of respondently conditioned stimulus function in accordance with arbitrarily applicable relations. Journal of the Experimental Analysis of Behavior, 67, 275-301.

Roche, B., Barnes-Holmes, D., Smeets, P. M., Barnes-Homes, Y., \& McGeady, S. (2000). Contextual control over the derived transformation of discriminative and sexual arousal functions. The Psychological Record, 50, 267-291.

Serna, R. W., \& Pérez-González, L. A. (2003). An analysis of generalized contextual control of conditional discriminations. Journal of the Experimental Analysis of Behavior, 79, 383-393.

Sidman, M. (1986). Functional analysis of emergent verbal classes. In T. Thompson \& M. D. Zeiler (Eds.), Analysis and integration of behavioral units (pp. 213-245). Hillsdale, NJ: Erlbaum.

Sidman, M. (1994). Equivalence relations and behavior: A research story. Boston, MA: Authors Cooperative.

Sidman, M. (2000). Equivalence relations and the reinforcement contingency. Journal of the Experimental Analysis of Behavior, 74, 127 146.

Steele, D., \& Hayes, S. C. (1991). Stimulus equivalence and arbitrarily applicable relational responding. Journal of the Experimental Analysis of Behavior, 56, 519-555.

Stewart, I., Hooper, N., Walsh, P., O'Keefe, R., Joyce, R., \& McHugh, L. (2015). Transformation of thought suppression functions via same and opposite relations. The Psychological Record, 65, 375-399.

Stromer, R., \& Osborne, J. G. (1982). Control of adolescents' arbitrary matching-to-sample by positive and negative stimulus relations. Journal of the Experimental Analysis of Behavior, 37, 329-348.

Tonneau, F. (2001). Equivalence relations: A critical analysis. European Journal of Behavior Analysis, 2, 1-33.

Whelan, R., \& Barnes-Holmes, D. (2004). The transformation of consequential functions in accordance with the relational frames of same and opposite. Journal of the Experimental Analysis of Behavior, 82, 177-195. 